\title{
Cross-cultural Research on the Creativity of Elementary School Students in Korea and Australia
}

\author{
Lee Kyunghwa ${ }^{1, *}$, Yang Hyejin ${ }^{2}$ \\ ${ }^{1}$ Department of Lifelong Education, Soongsil University, South Korea \\ ${ }^{2}$ Department of Architecture, Soongsil University, South Korea
}

Copyright $\bigcirc 2016$ by authors, all rights reserved. Authors agree that this article remains permanently open access under the terms of the Creative Commons Attribution License 4.0 International License

\begin{abstract}
The purpose of this study was to understand cultural differences and similarities in children's creative characteristics in Korea and Australia. In this cross-cultural research, the Integrative Creativity Test (K-ICT, [13]) with identified validity and reliability for measuring elementary school students' creative ability and creative personality, was taken by 187 participants consisting of 96 students $\left(4^{\text {th }}\right.$ grade to $6^{\text {th }}$ grade elementary school students) from Korea and 91 students $\left(4^{\text {th }}\right.$ grade to $6^{\text {th }}$ grade elementary school students) from Australia. Through the results of the creativity test, it showed both similarity and diversity according to three school grades and gender in Korean and Australian students. In integrative creativity and creative thinking ability, a significant difference was showed $(\mathrm{p}<.05)$ between the two countries but no difference in creative personality was found $(p>.05)$. The results of gender comparison were very diverse. In the case of girls, sensitive thinking and elaboration in Korean students was better than that of Australia students. However, task commitment and problem solving leadership of Australian students was better than those of Korean students. No significant difference was observed between $6^{\text {th }}$ graders in creativity. Korean $5^{\text {th }}$ grader's creative thinking ability was better than that of Australian students. But the $4^{\text {th }}$ grader of Korea student's creativity was better than that of Australian students. Hopefully, this research will help us to contribute to an understanding of cultural differences and similarities of student's creative characteristics in Korea and Australia.
\end{abstract}

Keywords Comparative Study, Creativity, Creative Thinking Ability, Creative Personality, AKC

\section{Introduction}

People engage in unique thinking because of an intrinsic desire to find new solutions and strategies to resolve either familiar or new challenges. This is what we called creativity. Especially the life in 21 st century is characterized by uncertainties because of social, economic, and technological changes in the world. So the next generation will need experiences and expertise in various fields that are not even known yet. The ability to resolve problems creatively enhances constructive and adaptive behavior in these new and demanding environments. Thus, problem solving skills and creativity are crucial for students who are growing up in these fast-changing and dynamic environments.

The current society we are living in requires creative thinking more than before. Because the concept of creativity is crucial for not only offering developmental successes to a person but also providing innovative chances to a country in the form of intellectual and information-oriented society. According to this fact, the word of creativity, this was usually mentioned in science and artistic circles in the past, was mentioned widely in various areas of our life such as enterprises, sports, and even in the daily experiences. With growing interests in the field of creativity in worldwide, the Republic of Korea has also paid more attention to the developmental aspects of creativity. With growing interests in the field of creativity worldwide, the Republic of Korea has paid more attention to the developmental aspects and education of creativity. Especially the Korea Ministry of Education presented the 'Creativity and Personality Educational Policy' in 2009 and supported creativity education to improve creativity and creative problem solving nation widely. As a part of this education, Lee insisted that creative thinking techniques can extend creative thinking and suggested a way to utilize the 50 kinds of creative thinking techniques in the curriculums [12].

Since Guilford addressed the definition of creativity in the 1950 s, various other definitions have been presented in the academic world. Psychologists have considered creativity as a trait of character, creative process, creative environment, and inventive product. Researchers went on to say that when the traits of character, process, environment, and product interact with each other, creativity is likely to be stirred or mobilized [6]. The concept of creativity has defined by various by scholars. Lee suggested that 
creativity is a factor comprised of personal ability and personality [9]. It could produce new and unique forms of personal ability and personality over developmental periods. Lee created the "Volcano model" as a way of understanding and measuring the creativity integrative [9]. The model showed various types of creativities along with the subject domains which are results of the links between abilities (e.g., intelligence, thinking ability, perception) and creative personality (e.g., motivation that based on developing personal environments). It also emphasized that the socio-cultural environment is crucial in developing creative traits. The functions played in creative thinking are factors such as sensitivity, diversity, fluency, flexibility, originality, elaboration, and imagination.

Over the past two decades, most theorists have defined creativity as the development of ideas about products, practices, services or procedures that are novel and potentially useful to the organization [1]. Although there is no agreement on definitions of creativity, different definitions agree on originality and usefulness as the two most important characteristics of the creative ideas or products [16]. On the other hand, it seems that creativity can be improved [4] and the effectiveness of the creativity training programs on different populations has been investigated ([2]; [8]). Also creativity, innovation, and ways to enhance them in organizational settings have been studied ([1]; [11]; [3]; [14]). Ideas are considered novel if they are unique relative to other ideas currently available in the organization.

Though gradually the interests of creativity increase in the world until now, there were not enough comparative studies for creativity. However, since the society such as Korea is heading toward a more globalized one, we must now know the traits of other country to understand ourselves better. If we know the developmental traits of children, we are able to support each other in developing educational program and measuring students' abilities more reliably at the national level. In order to understand creativity, there needs to be a series of research projects conducted in various related areas.

On the other hand, creativity has been defined that it is the personal ability producing novel and appropriate means ([15]). In this definition, there may be a question why it is novel and appropriate. Socio-cultural contexts affect the development of creativity and should, therefore, be included in the study of creativity. Accepting this socio-cultural point of view, it might be necessary to have comparative studies. According to the previous studies of the development of creativity, the tendency of development in childhood is not consistent. The study of discontinuity of developing creative traits might be related with cultural and social condition. However, to make sure this is the case, one needs to be confident about the test implementations and measures before taking a looking at developmental aspects. Most of the existing tests implementation occurred abroad and rarely reflected Korean cultural values. Furthermore, there was not enough comparative study using test measures of other countries' children, which the test implementation developed in Korea. Lee ([10], [13]) constructed "Integrated Individual Creativity Test: For preschooler and elementary school children." This test is culturally sensitive and standardized for Korean children. This test allows us to take a look at developing trends of the childhood, and how the creativity is different by the social and culture difference. This may be used as a basis for comparing children from two different cultures such as Australia and Korea in this case, how cultural differences would be reflected upon the test.

This study would help to understand culture differences of Australia and Korea. Two countries are located in Asia-Pacific region, but there are cultural differences between western and eastern. Culture is the general expression of humanity, the expression of its creativity. Culture is linked to meaning, knowledge, talents, industries, civilization and values. Culture-based creativity is a fundamental means for industry and policy decision makers to adopt and implement more user-centered strategies. By the importance of perspective that socio-cultural contexts affect the development of creativity and reflect the results of creativity tests [10], this cross-cultural research in Korea and Australia was progressed using the Korea integrative creativity test(K-ICT) developed by Lee [13]. The creativity test was developed and validated a Korean version of a creativity test and translated into English. It was tested children's creativity in Korea and Australia using this tool for cross-cultural research. Until now, there have not enough been comparative studies of creativity. If we know the developmental traits of children, we are able to support each other in developing educational programs of AKC (Australia and Korea Connection) and measuring students' abilities more reliably at the national level in Korea. The purpose of this study is to understand culture differences and similarities of children's creative characteristics between Korea and Australia, and also to contribute to the future cross-cultural studies with other countries in this field.

\section{Materials and Methods}

\subsection{Participants}

187 participants who consisted of 96 students $\left(4^{\text {th }}\right.$ grade to $6^{\text {th }}$ grade elementary school students) from Korea (Seoul \& Anyang) and 91 students $\left(4^{\text {th }}\right.$ grade to $6^{\text {th }}$ grade elementary school students) from Australia (Sydney \& Armidale) were sampled in order to investigate the developmental characteristics of creativity. The subject children were participated in AKC (Australia and Korea Connection) program that was tele collaboration learning using video-conferencing. 


\subsection{Instrumentation}

The 'Korea Integrative Creativity Test (K-ICT) for elementary and secondary school students' was developed by Lee [13] reflects the basis of studies [10], [11], and involves two domains - language and drawing. The K-ICT can measure creative thinking ability and creative personality at the same time, in which creative thinking ability involves fluency, flexibility, originality, imagination, sensitivity (sensitive thinking), and elaboration. The creative personality test consists of 30 items (5 Likert scale) and has no time limitation. The sub-factors of creativity in the creative personality consist of curiosity, sensitive personality, task commitment independence/adventure, humor and problem-solving leadership. The reliability of K-ICT is .94 (Cronbach's alpha).

For the purpose of this study, English version of K-ICT was used. The English version of K-ICT was identified the content validity by an American professor and Australian professors, and it was already used in the U.S.A. and Turkey.

\section{Results}

It assumed there would be diversity and similarities in creativity (creative ability and creative personality) by two domains of creativity (language, and drawing) throughout developmental stages (elementary grades 3rd to 6th), and culture in children of Korea and Australia. The results were presented according to variables as follows.

\subsection{Comparison in Creativity between Korea and Australia}

To know the differences and similarities of two countries, the results of K-ICT were compared with t-test using SPSS Win 21.0. The mean comparison was presented in Table 1.

According to comparison of the creativity test, it showed both similarity and diversity in Korean and Australian students. In integrative creativity and creative thinking ability, a significant difference was showed $(\mathrm{p}<.05)$ between the two countries but no significant difference in creative personality was identified $(\mathrm{p}>.05)$. Also significant difference was in sensitivity and elaboration of sub-factors of creative thinking ability and curiosity of creative personality (Table 1).

Table 1. Mean comparison of creativity between Korea and Australia (t-test)

\begin{tabular}{|c|c|c|c|c|c|c|}
\hline \multicolumn{5}{|c|}{ statistics } & \multirow{2}{*}{$t$} & \multirow{2}{*}{$p$} \\
\hline & & $N$ & $M$ & $S D$ & & \\
\hline \multirow{2}{*}{ fluency } & Australia & 91 & 15.93 & 6.73 & \multirow[t]{2}{*}{-.148} & \multirow[t]{2}{*}{.883} \\
\hline & Korea & 96 & 16.06 & 5.06 & & \\
\hline \multirow{2}{*}{ flexibility } & Australia & 91 & 4.08 & 2.36 & \multirow[t]{2}{*}{-1.463} & \multirow[t]{2}{*}{.145} \\
\hline & Korea & 96 & 4.56 & 2.18 & & \\
\hline \multirow{2}{*}{ originality } & Australia & 91 & 1.82 & 1.40 & \multirow[t]{2}{*}{-.787} & \multirow[t]{2}{*}{.432} \\
\hline & Korea & 96 & 2.00 & 1.64 & & \\
\hline \multirow{2}{*}{ imagination } & Australia & 91 & 4.12 & 1.36 & \multirow[t]{2}{*}{-1.528} & \multirow[t]{2}{*}{.128} \\
\hline & Korea & 96 & 4.43 & 1.38 & & \\
\hline \multirow{2}{*}{ sensitivity } & Australia & 91 & 3.93 & 1.93 & \multirow[t]{2}{*}{-5.551} & \multirow[t]{2}{*}{.000} \\
\hline & Korea & 96 & 5.48 & 1.87 & & \\
\hline \multirow{2}{*}{ elaboration } & Australia & 91 & 4.36 & 2.51 & \multirow[t]{2}{*}{-4.945} & \multirow[t]{2}{*}{.000} \\
\hline & Korea & 96 & 6.70 & 3.78 & & \\
\hline \multirow{2}{*}{ creative ability } & Australia & 91 & 34.25 & 11.19 & \multirow[t]{2}{*}{-3.258} & \multirow[t]{2}{*}{.001} \\
\hline & Korea & 96 & 39.23 & 9.68 & & \\
\hline \multirow{2}{*}{ curiosity } & Australia & 91 & 3.42 & .60 & \multirow[t]{2}{*}{-3.315} & \multirow[t]{2}{*}{.001} \\
\hline & Korea & 96 & 3.82 & .98 & & \\
\hline \multirow{2}{*}{ sensitive personality } & Australia & 91 & 3.47 & .60 & \multirow[t]{2}{*}{.102} & \multirow[t]{2}{*}{.919} \\
\hline & Korea & 96 & 3.46 & .70 & & \\
\hline to lo & Australia & 91 & 3.49 & .61 & -.447 & .655 \\
\hline lask commintment & Korea & 96 & 3.54 & .76 & & \\
\hline humor & Australia & 91 & 3.53 & .72 & .470 & .639 \\
\hline numor & Korea & 96 & 3.47 & .86 & & \\
\hline indenendent/odventure & Australia & 91 & 3.70 & .64 & 1.151 & .251 \\
\hline maependent /adventure & Korea & 96 & 3.57 & .84 & & \\
\hline problem- & Australia & 91 & 3.58 & .59 & .807 & .421 \\
\hline solving leadership & Korea & 96 & 3.50 & .79 & & \\
\hline creative nersonality & Australia & 91 & 3.53 & .48 & -.332 & .740 \\
\hline creative personamy & Korea & 96 & 3.56 & .66 & & \\
\hline oreotivity & Australia & 91 & 37.78 & 11.35 & -3.223 & .001 \\
\hline creativity & Korea & 96 & 42.79 & 9.87 & & \\
\hline
\end{tabular}




\subsection{Comparison of Grade between Korea and Australia}

Mean comparison of $4^{\text {th }}, 5^{\text {th }}, 6^{\text {th }}$ grade student's creativity between Korea and Australia by t-test was presented in Table 2, Table 3, Table 4.

According to comparison of $4^{\text {th }}, 5^{\text {th }}, 6^{\text {th }}$ grade student's creativity between Korea and Australia it showed both similarity and differences in Korean and Australian students. $4^{\text {th }}$ and $5^{\text {th }}$ students showed significant difference in integrative creativity and creative thinking ability between the two countries $(\mathrm{p}<.05)$, and also significant difference was in sensitivity and elaboration of sub-factors of creative thinking ability and curiosity of creative personality.

$4^{\text {th }}$ students showed differences in fluency of creative thinking ability and task commitment of creative personality, and $6^{\text {th }}$ students showed differences $(\mathrm{p}<.05)$ only in sensitivity and elaboration of sub-factors of creative thinking ability and humor of creative personality between the two countries (Table 2, 3, 4).

It was so interesting that there was different by graders.

Table 2. Mean comparison of creativity of the $4^{\text {th }}$ grade between Korea and Australia (t-test)

\begin{tabular}{|c|c|c|c|c|c|c|}
\hline \multicolumn{5}{|c|}{ statistics } & \multirow{2}{*}{$t$} & \multirow[b]{2}{*}{$p$} \\
\hline & & $N$ & $M$ & $S D$ & & \\
\hline \multirow{2}{*}{ fluency } & Australia & 11 & 10.73 & 8.19 & \multirow[t]{2}{*}{-2.204} & \multirow[t]{2}{*}{.039} \\
\hline & Korea & 11 & 16.82 & 4.12 & & \\
\hline \multirow{2}{*}{ flexibility } & Australia & 11 & 2.18 & 1.72 & \multirow[t]{2}{*}{-1.862} & \multirow[t]{2}{*}{.077} \\
\hline & Korea & 11 & 3.73 & 2.15 & & \\
\hline \multirow{2}{*}{ originality } & Australia & 11 & 1.27 & 1.27 & \multirow[t]{2}{*}{.164} & \multirow[t]{2}{*}{.871} \\
\hline & Korea & 11 & 1.18 & 1.33 & & \\
\hline \multirow{2}{*}{ imagination } & Australia & 11 & 4.09 & 1.58 & \multirow[t]{2}{*}{-1.017} & \multirow[t]{2}{*}{.321} \\
\hline & Korea & 11 & 4.73 & 1.35 & & \\
\hline \multirow{2}{*}{ sensitivity } & Australia & 11 & 2.55 & 1.57 & \multirow[t]{2}{*}{-3.357} & \multirow[t]{2}{*}{.003} \\
\hline & Korea & 11 & 5.64 & 2.62 & & \\
\hline \multirow{2}{*}{ elaboration } & Australia & 11 & 3.73 & 1.35 & \multirow[t]{2}{*}{-2.923} & \multirow[t]{2}{*}{.008} \\
\hline & Korea & 11 & 8.09 & 4.76 & & \\
\hline \multirow{2}{*}{ creative ability } & Australia & 11 & 24.55 & 12.29 & \multirow[t]{2}{*}{-3.293} & \multirow[t]{2}{*}{.004} \\
\hline & Korea & 11 & 40.18 & 9.85 & & \\
\hline \multirow{2}{*}{ curiosity } & Australia & 11 & 3.27 & .73 & \multirow[t]{2}{*}{-2.282} & \multirow[t]{2}{*}{.034} \\
\hline & Korea & 11 & 3.98 & .72 & & \\
\hline \multirow{2}{*}{ Sensitive personality } & Australia & 11 & 3.36 & .39 & \multirow[t]{2}{*}{-2.055} & \multirow[t]{2}{*}{.053} \\
\hline & Korea & 11 & 3.82 & .62 & & \\
\hline 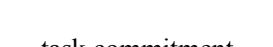 & Australia & 11 & 3.15 & .53 & -3.247 & .004 \\
\hline 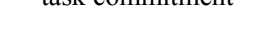 & Korea & 11 & 3.89 & .55 & & \\
\hline 1humart & Australia & 11 & 3.25 & .47 & -1.698 & .105 \\
\hline Humint & Korea & 11 & 3.67 & .67 & & \\
\hline ind nondont / drontu & Australia & 11 & 3.53 & .56 & -1.989 & .061 \\
\hline Н18 & Korea & 11 & 4.02 & .60 & & \\
\hline problem-solving & Australia & 11 & 3.64 & .61 & -.771 & .449 \\
\hline leadership & Korea & 11 & 3.87 & .82 & & \\
\hline 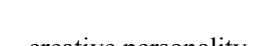 & Australia & 11 & 3.37 & .47 & -2.384 & .027 \\
\hline 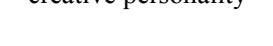 & Korea & 11 & 3.88 & .53 & & \\
\hline oreotivity & Australia & 11 & 27.91 & 12.36 & -3.352 & .003 \\
\hline Eivativity & Korea & 11 & 44.06 & 10.12 & & \\
\hline
\end{tabular}


Table 3. Mean comparison of creativity of the $5^{\text {th }}$ grade between Korea and Australia (t-test)

\begin{tabular}{|c|c|c|c|c|c|c|}
\hline \multicolumn{5}{|c|}{ statistics } & \multirow{2}{*}{$t$} & \multirow{2}{*}{$p$} \\
\hline & & $N$ & $M$ & $S D$ & & \\
\hline \multirow{2}{*}{ fluency } & Australia & 40 & 15.25 & 5.75 & \multirow[t]{2}{*}{-.613} & \multirow[t]{2}{*}{.541} \\
\hline & Korea & 40 & 16.00 & 5.16 & & \\
\hline \multirow{2}{*}{ flexibility } & Australia & 40 & 4.40 & 2.25 & \multirow[t]{2}{*}{-1.314} & \multirow[t]{2}{*}{.193} \\
\hline & Korea & 40 & 5.05 & 2.17 & & \\
\hline \multirow{2}{*}{ originality } & Australia & 40 & 2.03 & 1.49 & \multirow[t]{2}{*}{-1.970} & \multirow[t]{2}{*}{.052} \\
\hline & Korea & 40 & 2.73 & 1.68 & & \\
\hline \multirow{2}{*}{ imagination } & Australia & 40 & 4.30 & 1.34 & \multirow[t]{2}{*}{-.667} & \multirow[t]{2}{*}{.507} \\
\hline & Korea & 40 & 4.50 & 1.34 & & \\
\hline \multirow{2}{*}{ sensitivity } & Australia & 40 & 4.25 & 1.90 & \multirow[t]{2}{*}{-4.725} & \multirow[t]{2}{*}{.000} \\
\hline & Korea & 40 & 6.10 & 1.58 & & \\
\hline \multirow{2}{*}{ elaboration } & Australia & 40 & 4.15 & 2.14 & \multirow[t]{2}{*}{-2.438} & \multirow[t]{2}{*}{.017} \\
\hline & Korea & 40 & 5.58 & 3.01 & & \\
\hline \multirow{2}{*}{ creative ability } & Australia & 40 & 34.38 & 9.80 & \multirow[t]{2}{*}{-2.523} & \multirow[t]{2}{*}{.014} \\
\hline & Korea & 40 & 39.95 & 9.96 & & \\
\hline \multirow{2}{*}{ curiosity } & Australia & 40 & 3.42 & .55 & \multirow[t]{2}{*}{-2.838} & \multirow[t]{2}{*}{.006} \\
\hline & Korea & 40 & 3.99 & 1.16 & & \\
\hline \multirow{2}{*}{ sensitive personality } & Australia & 40 & 3.47 & .57 & \multirow[t]{2}{*}{.035} & \multirow[t]{2}{*}{.972} \\
\hline & Korea & 40 & 3.47 & .68 & & \\
\hline & Australia & 40 & 3.52 & .55 & -.070 & .945 \\
\hline 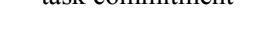 & Korea & 40 & 3.53 & .72 & & \\
\hline & Australia & 40 & 3.54 & .77 & -.757 & .451 \\
\hline 11010101 & Korea & 40 & 3.68 & .88 & & \\
\hline & Australia & 40 & 3.78 & .71 & .451 & .653 \\
\hline 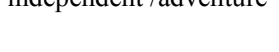 & Korea & 40 & 3.71 & .78 & & \\
\hline problem-solving & Australia & 40 & 3.55 & .67 & -.066 & .948 \\
\hline leadership & Korea & 40 & 3.56 & .69 & & \\
\hline & Australia & 40 & 3.54 & .47 & -.904 & .369 \\
\hline Cicative persomanty & Korea & 40 & 3.65 & .60 & & \\
\hline oreativity & Australia & 40 & 37.92 & 9.98 & -2.537 & .013 \\
\hline Eterivity & Korea & 40 & 43.60 & 10.06 & & \\
\hline
\end{tabular}


Table 4. Mean comparison of creativity of the $6^{\text {th }}$ grade between Korea and Australia (t-test)

\begin{tabular}{|c|c|c|c|c|c|c|}
\hline \multicolumn{5}{|c|}{ statistics } & \multirow{2}{*}{$t$} & \multirow{2}{*}{$p$} \\
\hline & & $N$ & $M$ & $S D$ & & \\
\hline \multirow{2}{*}{ fluency } & Australia & 40 & 18.05 & 6.45 & \multirow[t]{2}{*}{1.667} & \multirow[t]{2}{*}{.099} \\
\hline & Korea & 45 & 15.93 & 5.25 & & \\
\hline \multirow{2}{*}{ flexibility } & Australia & 40 & 4.28 & 2.43 & \multirow[t]{2}{*}{-.118} & \multirow[t]{2}{*}{.906} \\
\hline & Korea & 45 & 4.33 & 2.13 & & \\
\hline \multirow{2}{*}{ originality } & Australia & 40 & 1.78 & 1.31 & \multirow[t]{2}{*}{.732} & \multirow[t]{2}{*}{.466} \\
\hline & Korea & 45 & 1.56 & 1.44 & & \\
\hline \multirow{2}{*}{ imagination } & Australia & 40 & 3.95 & 1.32 & \multirow[t]{2}{*}{-1.126} & \multirow[t]{2}{*}{.263} \\
\hline & Korea & 45 & 4.29 & 1.44 & & \\
\hline \multirow{2}{*}{ sensitivity } & Australia & 40 & 4.00 & 1.92 & \multirow[t]{2}{*}{-2.225} & \multirow[t]{2}{*}{.029} \\
\hline & Korea & 45 & 4.89 & 1.76 & & \\
\hline \multirow{2}{*}{ elaboration } & Australia & 40 & 4.75 & 3.04 & \multirow[t]{2}{*}{-3.378} & \multirow[t]{2}{*}{.001} \\
\hline & Korea & 45 & 7.36 & 3.95 & & \\
\hline \multirow{2}{*}{ creative ability } & Australia & 40 & 36.80 & 10.99 & \multirow[t]{2}{*}{-.699} & \multirow[t]{2}{*}{.486} \\
\hline & Korea & 45 & 38.36 & 9.53 & & \\
\hline \multirow{2}{*}{ curiosity } & Australia & 40 & 3.48 & .61 & \multirow[t]{2}{*}{-.969} & \multirow[t]{2}{*}{.336} \\
\hline & Korea & 45 & 3.63 & .84 & & \\
\hline \multirow{2}{*}{ sensitive personality } & Australia & 40 & 3.50 & .68 & \multirow[t]{2}{*}{.855} & \multirow[t]{2}{*}{.395} \\
\hline & Korea & 45 & 3.36 & .72 & & \\
\hline t & Australia & 40 & 3.57 & .66 & .628 & .531 \\
\hline ТАЛ С & Korea & 45 & 3.46 & .83 & & \\
\hline humor & Australia & 40 & 3.60 & .71 & 2.063 & .042 \\
\hline 11010101 & Korea & 45 & 3.24 & .84 & & \\
\hline Findo ondo t l & Australia & 40 & 3.66 & .59 & 1.930 & .057 \\
\hline 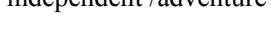 & Korea & 45 & 3.34 & .88 & & \\
\hline problem-solving & Australia & 40 & 3.60 & .50 & 1.609 & .111 \\
\hline leadership & Korea & 45 & 3.35 & .83 & & \\
\hline . & Australia & 40 & 3.56 & .49 & 1.228 & .223 \\
\hline Ciealive personianty & Korea & 45 & 3.40 & .71 & & \\
\hline . & Australia & 40 & 40.36 & 11.14 & -.614 & .541 \\
\hline creativity & Korea & 45 & 41.75 & 9.75 & & \\
\hline
\end{tabular}

\subsection{Comparison of gender between Korea and Australia}

Mean comparison of boys' and girls' creativity between Korea and Australia by t-test was presented in Table 5, Table 6 .

According to gender comparison of creativity between Korea and Australia, it showed interesting results rather than other variables. There were differences in boy's creativity showed significant difference in integrative creativity and creative thinking ability(flexibility, sensitivity, elaboration) between the two countries $(p<.05)$, and also significant difference in creative personality(curiosity, task commitment) was showed. But the results of girl's creativity were difference.

The sensitivity and elaboration of sub-factor of creative thinking ability of Korean girl was better than Australian girl's creativity. Unlike creative abilities, different results were obtained in the creative personality. The Australian girl's task commitment and problem solving leadership of creative personality was better than Korean girls' (Table 5, 6). 
Table 5. Mean comparison of creativity of boy between Korea and Australia (t-test)

\begin{tabular}{|c|c|c|c|c|c|c|}
\hline \multicolumn{5}{|c|}{ statistics } & \multirow{2}{*}{$t$} & \multirow{2}{*}{$p$} \\
\hline & & $N$ & $M$ & $S D$ & & \\
\hline \multirow{2}{*}{ fluency } & Australia & 46 & 15.00 & 6.70 & \multirow[t]{2}{*}{-.491} & \multirow[t]{2}{*}{.624} \\
\hline & Korea & 53 & 15.55 & 4.26 & & \\
\hline \multirow{2}{*}{ flexibility } & Australia & 46 & 3.39 & 2.09 & \multirow[t]{2}{*}{-2.641} & \multirow[t]{2}{*}{.010} \\
\hline & Korea & 53 & 4.51 & 2.11 & & \\
\hline \multirow{2}{*}{ originality } & Australia & 46 & 1.48 & 1.24 & \multirow[t]{2}{*}{-1.091} & \multirow[t]{2}{*}{.278} \\
\hline & Korea & 53 & 1.75 & 1.27 & & \\
\hline \multirow{2}{*}{ imagination } & Australia & 46 & 4.00 & 1.59 & \multirow[t]{2}{*}{-.565} & \multirow[t]{2}{*}{.573} \\
\hline & Korea & 53 & 4.17 & 1.40 & & \\
\hline \multirow{2}{*}{ sensitivity } & Australia & 46 & 3.46 & 1.79 & \multirow[t]{2}{*}{-5.168} & \multirow[t]{2}{*}{.000} \\
\hline & Korea & 53 & 5.36 & 1.86 & & \\
\hline \multirow{2}{*}{ elaboration } & Australia & 46 & 4.02 & 2.37 & \multirow[t]{2}{*}{-4.305} & \multirow[t]{2}{*}{.000} \\
\hline & Korea & 53 & 6.30 & 2.83 & & \\
\hline \multirow{2}{*}{ creative ability } & Australia & 46 & 31.35 & 10.30 & \multirow[t]{2}{*}{-3.464} & \multirow[t]{2}{*}{.001} \\
\hline & Korea & 53 & 37.64 & 7.73 & & \\
\hline \multirow{2}{*}{ curiosity } & Australia & 46 & 3.45 & .63 & \multirow[t]{2}{*}{-3.531} & \multirow[t]{2}{*}{.001} \\
\hline & Korea & 53 & 4.07 & 1.04 & & \\
\hline \multirow{2}{*}{ sensitive personality } & Australia & 46 & 3.47 & .60 & \multirow[t]{2}{*}{-1.649} & \multirow[t]{2}{*}{.102} \\
\hline & Korea & 53 & 3.67 & .64 & & \\
\hline th & Australia & 46 & 3.39 & .64 & -2.699 & .008 \\
\hline & Korea & 53 & 3.76 & .72 & & \\
\hline humor $\mathrm{s}+\mathrm{s}$ & Australia & 46 & 3.46 & .78 & -.903 & .369 \\
\hline & Korea & 53 & 3.60 & .80 & & \\
\hline & Australia & 46 & 3.70 & .64 & .439 & .662 \\
\hline 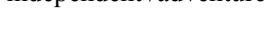 & Korea & 53 & 3.63 & .82 & & \\
\hline problem-solving & Australia & 46 & 3.51 & .62 & -1.462 & .147 \\
\hline leadership & Korea & 53 & 3.70 & .68 & & \\
\hline & Australia & 46 & 3.49 & .49 & -2.191 & .031 \\
\hline 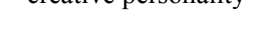 & Korea & 53 & 3.74 & .61 & & \\
\hline & Australia & 46 & 34.84 & 10.43 & -3.554 & .001 \\
\hline & Korea & 53 & 41.38 & 7.83 & & \\
\hline
\end{tabular}


Table 6. Mean comparison of creativity of girl between Korea and Australia (t-test)

\begin{tabular}{|c|c|c|c|c|c|c|}
\hline \multicolumn{5}{|c|}{ statistics } & \multirow{2}{*}{$t$} & \multirow{2}{*}{$p$} \\
\hline & & $N$ & $M$ & $S D$ & & \\
\hline \multirow{2}{*}{ fluency } & Australia & 45 & 16.89 & 6.70 & \multirow[t]{2}{*}{.142} & \multirow[t]{2}{*}{.887} \\
\hline & Korea & 43 & 16.70 & 5.89 & & \\
\hline \multirow{2}{*}{ flexibility } & Australia & 45 & 4.78 & 2.44 & \multirow[t]{2}{*}{.298} & \multirow[t]{2}{*}{.767} \\
\hline & Korea & 43 & 4.63 & 2.28 & & \\
\hline \multirow{2}{*}{ originality } & Australia & 45 & 2.18 & 1.47 & \multirow[t]{2}{*}{-.336} & \multirow[t]{2}{*}{.738} \\
\hline & Korea & 43 & 2.30 & 1.98 & & \\
\hline \multirow{2}{*}{ imagination } & Australia & 45 & 4.24 & 1.07 & \multirow[t]{2}{*}{-1.963} & \multirow[t]{2}{*}{.053} \\
\hline & Korea & 43 & 4.74 & 1.31 & & \\
\hline \multirow{2}{*}{ sensitivity } & Australia & 45 & 4.42 & 1.97 & \multirow[t]{2}{*}{-2.917} & \multirow[t]{2}{*}{.005} \\
\hline & Korea & 43 & 5.63 & 1.90 & & \\
\hline \multirow{2}{*}{ elaboration } & Australia & 45 & 4.71 & 2.63 & \multirow[t]{2}{*}{-3.072} & \multirow[t]{2}{*}{.003} \\
\hline & Korea & 43 & 7.19 & 4.69 & & \\
\hline \multirow{2}{*}{ creative ability } & Australia & 45 & 37.22 & 11.38 & \multirow[t]{2}{*}{-1.629} & \multirow[t]{2}{*}{.107} \\
\hline & Korea & 43 & 41.19 & 11.43 & & \\
\hline \multirow{2}{*}{ curiosity } & Australia & 45 & 3.40 & .57 & \multirow[t]{2}{*}{-.773} & \multirow[t]{2}{*}{.441} \\
\hline & Korea & 43 & 3.52 & .82 & & \\
\hline \multirow{2}{*}{ sensitive personality } & Australia & 45 & 3.47 & .61 & \multirow[t]{2}{*}{1.986} & \multirow[t]{2}{*}{.050} \\
\hline & Korea & 43 & 3.20 & .69 & & \\
\hline to a & Australia & 45 & 3.60 & .57 & 2.423 & .017 \\
\hline & Korea & 43 & 3.26 & .72 & & \\
\hline humor & Australia & 45 & 3.60 & .64 & 1.687 & .095 \\
\hline 114010101 & Korea & 43 & 3.32 & .92 & & \\
\hline -indono d n t & Australia & 45 & 3.69 & .64 & 1.246 & .216 \\
\hline 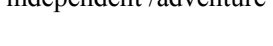 & Korea & 43 & 3.49 & .86 & & \\
\hline problem-solving & Australia & 45 & 3.65 & .55 & 2.716 & .008 \\
\hline leadership & Korea & 43 & 3.24 & .83 & & \\
\hline (a) t. & Australia & 45 & 3.57 & .47 & 1.875 & .064 \\
\hline 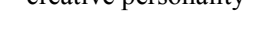 & Korea & 43 & 3.34 & .67 & & \\
\hline oreativity & Australia & 45 & 40.79 & 11.57 & -1.500 & .137 \\
\hline Givativity & Korea & 43 & 44.52 & 11.77 & & \\
\hline
\end{tabular}

\section{Conclusions}

The developmental trends and characteristics of elementary school student's creativity are one of the most interesting topics of discussion in educational and psychological fields. Through this study, the differences and similarities for each developmental stage and gender by culture were identified. The responses of the creativity test in integrative creativity, creative thinking ability and creative personality were very interesting in Korea and Australia children. The responses of the creativity test in two factors and sub-factors (creative thinking abilityfluency, flexibility, originality, elaboration, sensitive thinking; creative personality- curiosity, sensitive personality, task commitment, humor, independence, leadership) were showed the similarity and diversity according to three developmental stages and gender in Korea and Australia students. Normally the differences in the integrative creativity and creative thinking ability between two countries showed $(\mathrm{p}<.05)$, but the creative personality was very similar $(\mathrm{p}>.05)$. The results of gender comparison was very diverse, in the case of girl, the sensitive thinking and elaboration of Korea students was better than Australia students but task commitment and leadership of Australia students was better. And the result of graders comparison was different and in the difference of 
4th graders was distinct. There were very similar developmental traits between two countries, but according to the graders there was very interest difference showed. The difference of 6th grader's creativity was not significant, the 5th grader's creative thinking ability of Korean was better but the creative personality was somewhat different. But the 4th grader of Korea student's creativity was better than Australia students. Hopefully, this research will help us to contribute understand cultural differences and similarities of student's creative characteristics in Korea and Australia.

The results of this study will offer three significant meanings for educators and researchers as follows. Firstly, the developmental treats of elementary level students' creativity in both countries were identified through this study. Then, identifying these treats would help to understand cultural differences among Korea and other cultures to be studied in the future. There will be reciprocal benefits for both researchers in Korea and Australia. Secondly, it will help to develop the curriculum and teaching program for fostering the creativity for children from both countries. Thirdly, as not yet most of the existing tests (for young children and elementary, middle, high school students) developed and validated by Lee in Korea implemented to other countries [13]. Though the test, "Integrative Creativity Test: For elementary school children", developed in Korea was used to compare children from two different cultures of Korea and Australia.

Hopefully, this research is expected to help us understanding cultural differences and similarities of children's creative characteristics in Korea and Australia.

\section{Acknowledgements}

I am very grateful to editorial board of Universal Journal of Educational Research for suggestion of submission.

\section{REFERENCES}

[1] Amabile, T. M. Creativity and innovation in organizations (Vol. 5). Boston: Harvard Business School. 1996

[2] Benedek, M., Fink, A., \& Neubauer, A. C. Enhancement of ideational fluency by means of computer-based training. Creativity Research Journal, 18(3), 2006: 317-328.

[3] Birdi, K. A lighthouse in the desert? Evaluating the effects of creativity training on employee innovation. The Journal of Creative Behavior, 41(4), 2007: 249-270.

[4] Fasko, D. Education and creativity. Creativity research journal, 13(3-4), 2001: 317-327.

[5] Feldhusen, J. F. \& Treffinger, D. J. Creative thinking and problem solving in gifted education. Kendall/ Hunt Publishing co. 1986

[6] Feldhusen, J. F. A conception of creative thinking and creativity training. In S. G. Isaksen, M. C. Murdock, R. L. Firestien \& D. J. Treffinger (Eds.) Nurturing and developing creativity: Emergence of a discipline .Norwood, NJ: Ablex. 1993

[7] Guilford, J. P. The nature of human intelligence. New York: McGraw-Hill Book Co. 1967

[8] Kassim, H., Nicholas, H., \& Ng, W. Using a multimedia learning tool to improve creative performance. Thinking Skills and Creativity, 2014: 13, 9-19.

[9] Lee, K. Creative Ability and Creative Personality of 4, 5years old children, The Korean Journal of Educational Psychology, Vol. 16, No.3, 2002: 147-160.

[10] Lee, K. The Development Trends of Creative Thinking Ability and Creative Personality of Elementary School Children, The 15th Biennial Conference of the World Council for Gifted and Talented Children. 2003

[11] Lee, K. Effects of Future City Construction Project Instruction Using CPS on Creativity and Problem Solving Ability of Elementary School Children. Korean Journal of Educational Psychology, 20(2), 2006: 487-506.

[12] Lee, K. Creativity and Personality Curriculum: Inter Subject-matter. KOFAC. 2010

[13] Lee, K. Integrative Creativity Test, Seoul: Hakjisa. 2014

[14] Mumford, M. D. (Ed.). Handbook of organizational creativity. Academic Press. 2012

[15] Ochse, R. Before the gates of excellence: The determinant of creative genius. New York: Cambridge University Press. 1990

[16] Runco, M. A., \& Jaeger, G. J. The standard definition of creativity. Creativity Research Journal, 24(1), 2012: 92-96.

[17] Sternberg, R. J. (Ed.). The nature of creativity. New York: Cambridge University Press. 1988 